\title{
Integrable motion of two interacting curves, spin systems and the Manakov system
}

\author{
Akbota Myrzakul* and Ratbay Myrzakulov ${ }^{\dagger}$ \\ Eurasian International Center for Theoretical Physics and Department of General \\ \& Theoretical Physics, Eurasian National University, Astana 010008, Kazakhstan
}

\begin{abstract}
Integrable spin systems are an important subclass of integrable (soliton) nonlinear equations. They play important role in physics and mathematics. At present, many integrable spin systems were found and studied. They are related with the motion of 3-dimensional curves. In this paper, we consider a model of two moving interacting curves. Next, we find its integrable reduction related with some integrable coupled spin system. Then we show that this integrable coupled spin system is equivalent to the famous Manakov system.
\end{abstract}

\section{Introduction}

Among the integrable systems, the integrable spin systems in $1+1$ and $2+1$ dimensions play an important role in physics and mathematics 1]-15. In physics, they describe nonlinear dynamics of magnets. In differential geometry, they can reproduce some integrable classes of curves and surfaces [16-26]. The first and the most known representative of the integrable spin systems is the Heisenberg ferromagnetic equation (HFE) which has a form

$$
i A_{t}+\frac{1}{2}\left[A, A_{x x}\right]=0
$$

where $\mathbf{A}=\left(A_{1}, A_{2}, A_{3}\right)$ is a unit spin vector, $\mathbf{A}^{2}=1$ and

$$
A=\left(\begin{array}{cc}
A_{3} & A^{-} \\
A^{+} & -A_{3}
\end{array}\right), \quad A^{2}=I=\operatorname{diag}(1,1), \quad A^{ \pm}=A_{1} \pm i A_{2} .
$$

The HFE is the Lakshmanan equivalent [7] to the nonlinear Schrödinger equation

$$
i q_{t}+q_{x x}+2|q|^{2} q=0
$$

Also, it is well-known that these equations are gauge equivalent to each other [8]. At present, many integrable and nonintegrable spin systems were identified (see e.g. Refs. [27]-[40] and references therein). One of such spin systems is the Myrzakulov-LIII equation or shortly, the M-LIII equation 1. The M-LIII equation reads as [11]-35]

$$
i A_{t}+\frac{1}{2}\left[A, A_{x x}\right]+i u A_{x}=0,
$$

\footnotetext{
*Email: akbota.myrzakul@gmail.com

${ }^{\dagger}$ Email: rmyrzakulov@gmail.com

${ }^{1}$ Here LIII $\equiv 53$ so that M-LIII $\equiv$ M-53 and the M-LIII equation $\equiv$ the M-53 equation.
} 
where $u=u(t, x)$ is some real function (potential). The modified (inhomogeneous) M-LIII equation looks like

$$
i A_{t}+\frac{1}{2}\left[A, A_{x x}\right]+i u A_{x}+F=0,
$$

where $F$ is a matrix function. In this paper, we study the two-layer ("two-component") generalization of the modified M-LIII equation (1.5) or in short, the coupled M-LIII equation.

The paper is organized as follows. In Sec. 2, the coupled M-LIII equation is introduced. In Sec. 3, we derived the Lakshmanan equivalent counterpart of the M-LIII equation, namely, the Manakov system. In Sec. 4, we present two types of Lax representations of the coupled M-LIII equation. In Sec. 5, the relation between the solutions of the Manakov system and the M-LIII equation is established. The gauge equivalent counterpart of the Manakov system is presented in Sec. 6. The relation between solutions of the coupled M-LIII equation and the $\Gamma$-spin system is considered in Sec. 7. At last, Sec. 8 is devoted to Conclusions.

\section{The coupled M-LIII equation}

Consider two spin vectors $\mathbf{A}=\left(A_{1}, A_{2}, A_{3}\right)$ and $\mathbf{B}=\left(B_{1}, B_{2}, B_{3}\right)$, where $\mathbf{A}^{2}=\mathbf{B}^{2}=1$. Let these spin vectors satisfy the coupled Myrzakulov-LIII equation or the 2-layer Myrzakulov-LIII equation of the form 39$]-46$ ]

$$
\begin{aligned}
& i A_{t}+\frac{1}{2}\left[A, A_{x x}\right]+i u_{1} A_{x}+F=0, \\
& i B_{t}+\frac{1}{2}\left[B, B_{x x}\right]+i u_{2} B_{x}+E=0 .
\end{aligned}
$$

Here $u_{k}$ are real functions, $F$ and $E$ are matrix functions, $B$ is the matrix form of the $\mathbf{B}$ (second spin vector)

$$
B=\left(\begin{array}{cc}
B_{3} & B^{-} \\
B^{+} & -B_{3}
\end{array}\right), \quad F=\left(\begin{array}{cc}
F_{3} & F^{-} \\
F^{+} & -F_{3}
\end{array}\right), \quad E=\left(\begin{array}{cc}
E_{3} & E^{-} \\
E^{+} & -E_{3}
\end{array}\right),
$$

where $B^{ \pm}=B_{1} \pm i B_{2}, \quad B^{2}=I, \quad F^{ \pm}=F_{1} \pm i F_{2}, \quad E^{ \pm}=E_{1} \pm i E_{2}$. We now introduce two complex functions $u$ and $v$ as

$$
u=\frac{A^{+}}{1+A_{3}}, \quad v=\frac{B^{+}}{1+B_{3}} .
$$

Then these functions satisfy the following set of equations

$$
\begin{aligned}
& i u_{t}-u_{x x}+\frac{2 u^{*} u_{x}^{2}}{1+|u|^{2}}=F^{\prime}, \\
& i v_{t}-v_{x x}+\frac{2 v^{*} v_{x}^{2}}{1+|v|^{2}}=E^{\prime},
\end{aligned}
$$

where $F^{\prime}$ and $E^{\prime}$ are some complex functions

$$
\begin{aligned}
& F^{\prime}=F^{\prime}\left(u, v, u_{x}, v_{x}, \ldots\right), \\
& E^{\prime}=E^{\prime}\left(u, v, u_{x}, v_{x}, \ldots\right) .
\end{aligned}
$$

In this paper, we assume that $F$ and $E$ have the form

$$
F=v_{1}\left[\sigma_{3}, A\right], \quad E=v_{2}\left[\sigma_{3}, B\right],
$$

where $v_{j}$ are some real functions (potentials). Then the coupled M-LIII equation (2.1)-(2.2) takes the form

$$
\begin{aligned}
i A_{t}+\frac{1}{2}\left[A, A_{x x}\right]+i u_{1} A_{x}+v_{1}\left[\sigma_{3}, A\right] & =0 \\
i B_{t}+\frac{1}{2}\left[B, B_{x x}\right]+i u_{2} B_{x}+v_{2}\left[\sigma_{3}, B\right] & =0 .
\end{aligned}
$$


Here $u_{j}$ and $v_{j}$ are coupling potentials and have the following forms

$$
\begin{aligned}
& u_{1}=\frac{i\left(\bar{Z} B^{-}-Z B^{+}\right)}{W\left(1+B_{3}\right)} \\
& v_{1}=-\frac{|Z|^{2}}{2 W\left(1+A_{3}\right)^{2}} \\
& u_{2}=\frac{i\left(\bar{R} A^{-}-R A^{+}\right)\left(1+B_{3}\right)}{W\left(1+A_{3}\right)^{2}} \\
& v_{2}=-\frac{|R|^{2}}{2 W\left(1+A_{3}\right)^{3}},
\end{aligned}
$$

where

$$
\begin{aligned}
W & =2+\frac{\left(1+A_{3}\right)\left(1-B_{3}\right)}{1+B_{3}}, \\
R & =W A_{x}^{-}-M A^{-}, \\
Z & =W\left[\left(1+A_{3}\right)\left(1+B_{3}\right)^{-1} B^{-}\right]_{x}-M\left[\left(1+A_{3}\right)\left(1+B_{3}\right)^{-1} B^{-}\right], \\
M & =A_{3 x}+\frac{A^{+} A_{x}^{-}}{1+A_{3}}+\frac{A_{3 x}\left(1-B_{3}\right)}{1+B_{3}}+\frac{\left(1+A_{3}\right) B^{+} B_{x}^{-}}{\left(1+B_{3}\right)^{2}}-\frac{\left(1+A_{3}\right)\left(1-B_{3}\right) B_{3 x}}{\left(1+B_{3}\right)^{2}} .
\end{aligned}
$$

In components, the 2-layer M-LIII equation (2.10)-(2.11) reads as

$$
\begin{aligned}
i A_{t}^{+}+\left(A^{+} A_{3 x x}-A_{x x}^{+} A_{3}\right)+i u_{1} A_{x}^{+}-2 v_{1} A^{+} & =0, \\
i A_{t}^{-}-\left(A^{-} A_{3 x x}-A_{x x}^{-} A_{3}\right)+i u_{1} A_{x}^{-}+2 v_{1} A^{-} & =0, \\
i A_{3 t}+\frac{1}{2}\left(A^{-} A_{x x}^{+}-A_{x x}^{-} A^{+}\right)+i u_{1} A_{3 x} & =0, \\
i B_{t}^{+}+\left(B^{+} B_{3 x x}-B_{x x}^{+} B_{3}\right)+i u_{2} B_{x}^{+}-2 v_{2} B^{+} & =0, \\
i B_{t}^{-}-\left(B^{-} B_{3 x x}-B_{x x}^{-} B_{3}\right)+i u_{2} B_{x}^{-}+2 v_{2} B^{-} & =0, \\
i B_{3 t}+\frac{1}{2}\left(B^{-} B_{x x}^{+}-B_{x x}^{-} B^{+}\right)+i u_{2} B_{3 x} & =0
\end{aligned}
$$

or

$$
\begin{aligned}
A_{1 t}+A_{2} A_{3 x x}-A_{2 x x} A_{3}+u_{1} A_{1 x}-2 v_{1} A_{2} & =0, \\
A_{2 t}+A_{3} A_{1 x x}-A_{3 x x} A_{1}+u_{1} A_{2 x}-2 v_{1} A_{1} & =0, \\
A_{3 t}+A_{1} A_{2 x x}-A_{1 x x} A_{2}+u_{1} A_{3 x} & =0, \\
B_{1 t}+B_{2} B_{3 x x}-B_{2 x x} B_{3}+u_{2} B_{1 x}-2 v_{2} B_{2} & =0, \\
B_{2 t}+B_{3} B_{1 x x}-B_{3 x x} B_{1}+u_{2} B_{2 x}-2 v_{2} B_{1} & =0, \\
B_{3 t}+B_{1} B_{2 x x}-B_{1 x x} B_{2}+u_{2} B_{3 x} & =0 .
\end{aligned}
$$

\section{Lakshmanan equivalent counterpart of the coupled M- LIII equation}

In this section, we present the Lakshmanan equivalent counterpart of the coupled M-LIII equation (2.10)-(2.11). To do that, let us rewrite the 2-layer M-LIII equation (2.10)-(2.11) in the vector form as $39-46$

$$
\begin{aligned}
\mathbf{A}_{t}+\mathbf{A} \wedge \mathbf{A}_{x x}+u_{1} \mathbf{A}_{x}+2 v_{1} \mathbf{H} \wedge \mathbf{A} & =0 \\
\mathbf{B}_{t}+\mathbf{B} \wedge \mathbf{B}_{x x}+u_{2} \mathbf{B}_{x}+2 v_{2} \mathbf{H} \wedge \mathbf{B} & =0
\end{aligned}
$$

where $\mathbf{H}=(0,0,1)$ is the constant magnetic field. Now we consider two interacting 3-dimensional curves in $R^{n}$. These curves are given by the following two basic vectors $\mathbf{e}_{k}$ and $\mathbf{l}_{k}$. The motion of these curves is defined by the following equations

$$
\left(\begin{array}{l}
\mathbf{e}_{1} \\
\mathbf{e}_{2} \\
\mathbf{e}_{3}
\end{array}\right)_{x}=C\left(\begin{array}{l}
\mathbf{e}_{1} \\
\mathbf{e}_{2} \\
\mathbf{e}_{3}
\end{array}\right), \quad\left(\begin{array}{l}
\mathbf{e}_{1} \\
\mathbf{e}_{2} \\
\mathbf{e}_{3}
\end{array}\right)_{t}=D\left(\begin{array}{l}
\mathbf{e}_{1} \\
\mathbf{e}_{2} \\
\mathbf{e}_{3}
\end{array}\right)
$$


and

$$
\left(\begin{array}{l}
\mathbf{l}_{1} \\
\mathbf{l}_{2} \\
\mathbf{l}_{3}
\end{array}\right)_{x}=L\left(\begin{array}{l}
\mathbf{l}_{1} \\
\mathbf{l}_{2} \\
\mathbf{l}_{3}
\end{array}\right), \quad\left(\begin{array}{l}
\mathbf{l}_{1} \\
\mathbf{l}_{2} \\
\mathbf{l}_{3}
\end{array}\right)_{t}=N\left(\begin{array}{l}
\mathbf{l}_{1} \\
\mathbf{l}_{2} \\
\mathbf{l}_{3}
\end{array}\right) .
$$

Here $\mathbf{e}_{1}, \mathbf{e}_{2}$ and $\mathbf{e}_{3}$ are the unit tangent, normal and binormal vectors respectively to the first curve, $\mathbf{l}_{1}, \mathbf{l}_{2}$ and $\mathbf{l}_{3}$ are the unit tangent, normal and binormal vectors respectively to the second curve, $x$ is the arclength parametrising these both curves. The matrices $C, D, L, N$ are given by

$$
\begin{aligned}
C & =\left(\begin{array}{ccc}
0 & k_{1} & 0 \\
-k_{1} & 0 & \tau_{1} \\
0 & -\tau_{1} & 0
\end{array}\right), \quad G=\left(\begin{array}{ccc}
0 & \omega_{3} & -\omega_{2} \\
-\omega_{3} & 0 & \omega_{1} \\
\omega_{2} & -\omega_{1} & 0
\end{array}\right), \\
L & =\left(\begin{array}{ccc}
0 & k_{2} & 0 \\
-k_{2} & 0 & \tau_{2} \\
0 & -\tau_{2} & 0
\end{array}\right), \quad N=\left(\begin{array}{ccc}
0 & \theta_{3} & -\theta_{2} \\
-\theta_{3} & 0 & \theta_{1} \\
\theta_{2} & -\theta_{1} & 0
\end{array}\right) .
\end{aligned}
$$

For the curvatures and torsions of curves we obtain

$$
\begin{aligned}
& k_{1}=\sqrt{\mathbf{e}_{1 x}^{2}}, \quad \tau_{1}=\frac{\mathbf{e}_{1} \cdot\left(\mathbf{e}_{1 x} \wedge \mathbf{e}_{1 x x}\right)}{\mathbf{e}_{1 x}^{2}}, \\
& k_{2}=\sqrt{\mathbf{l}_{1 x}^{2}}, \quad \tau_{2}=\frac{\mathbf{l}_{1} \cdot\left(\mathbf{l}_{1 x} \wedge \mathbf{l}_{1 x x}\right)}{\mathbf{l}_{1 x}^{2}} .
\end{aligned}
$$

The equations (3.3) and (3.4) are compatible if

$$
\begin{aligned}
& C_{t}-G_{x}+[C, G]=0, \\
& L_{t}-N_{x}+[L, N]=0,
\end{aligned}
$$

respectively. In elements these equations take the form

$$
\begin{aligned}
k_{1 t} & =\omega_{3 x}+\tau_{1} \omega_{2}, \\
\tau_{1 t} & =\omega_{1 x}-k_{1} \omega_{2}, \\
\omega_{2 x} & =\tau_{1} \omega_{3}-k_{1} \omega_{1}
\end{aligned}
$$

and

$$
\begin{aligned}
k_{2 t} & =\theta_{3 x}+\tau_{2} \theta_{2}, \\
\tau_{2 t} & =\theta_{1 x}-k_{2} \theta_{2}, \\
\theta_{2 x} & =\tau_{2} \theta_{3}-k_{2} \theta_{1},
\end{aligned}
$$

respectively. Our next step is the following identifications:

$$
\mathbf{A} \equiv \mathbf{e}_{1}, \quad \mathbf{B} \equiv \mathbf{l}_{1} .
$$

We also assume that

$$
\mathbf{F}=F_{1} \mathbf{e}_{1}+F_{2} \mathbf{e}_{2}+F_{3} \mathbf{e}_{3}, \quad \mathbf{E}=E_{1} \mathbf{l}_{1}+E_{2} \mathbf{l}_{2}+E_{3} \mathbf{l}_{3},
$$

where

$$
\mathbf{F}=2 v_{1} \mathbf{H} \wedge \mathbf{A}, \quad \mathbf{E}=2 v_{2} \mathbf{H} \wedge \mathbf{B} .
$$

Then we obtain

$$
\begin{aligned}
k_{1} & =\sqrt{\mathbf{A}_{x}^{2}} \\
\tau_{1} & =\frac{\mathbf{A} \cdot\left(\mathbf{A}_{x} \wedge \mathbf{A}_{x x}\right)}{\mathbf{A}_{x}^{2}}, \\
k_{2} & =\sqrt{\mathbf{B}_{x}^{2}}, \\
\tau_{2} & =\frac{\mathbf{B} \cdot\left(\mathbf{B}_{x} \wedge \mathbf{B}_{x x}\right)}{\mathbf{B}_{x}^{2}}
\end{aligned}
$$


and

$$
\begin{aligned}
\omega_{1} & =-\frac{k_{1 x x}+F_{2} \tau_{1}+F_{3 x}}{k_{1}}+\left(\tau_{1}-u_{1}\right) \tau_{1}, \\
\omega_{2} & =k_{1 x}+F_{3}, \\
\omega_{3} & =k_{1}\left(\tau_{1}-u_{1}\right)-F_{2}, \\
\theta_{1} & =-\frac{k_{2 x x}+E_{2} \tau_{2}+E_{3 x}}{k_{2}}+\left(\tau_{2}-u_{2}\right) \tau_{2}, \\
\theta_{2} & =k_{2 x}+E_{3}, \\
\theta_{3} & =k_{2}\left(\tau_{2}-u_{2}\right)-E_{2}
\end{aligned}
$$

with

$$
F_{1}=E_{1}=0 .
$$

We now can write the equations for $k_{j}$ and $\tau_{j}$. They look like

$$
\begin{aligned}
k_{1 t} & =2 k_{1 x} \tau_{1}+k_{1} \tau_{1 x}-\left(u_{1} k_{1}\right)_{x}-F_{2 x}+F_{3} \tau_{1}, \\
\tau_{1 t} & =\left[-\frac{k_{1 x x}+F_{2} \tau_{1}+F_{3 x}}{k_{1}}+\left(\tau_{1}-u_{1}\right) \tau_{1}-\frac{1}{2} k_{1}^{2}\right]_{x}-F_{3} k_{1}, \\
k_{2 t} & =2 k_{2 x} \tau_{2}+k_{2} \tau_{2 x}-\left(u_{2} k_{2}\right)_{x}-E_{2 x}+E_{3} \tau_{2}, \\
\tau_{2 t} & =\left[-\frac{k_{2 x x}+E_{2} \tau_{2}+E_{3 x}}{k_{2}}+\left(\tau_{2}-u_{2}\right) \tau_{2}-\frac{1}{2} k_{2}^{2}\right]_{x}-E_{3} k_{2} .
\end{aligned}
$$

Let us now introduce new four real functions $\alpha_{j}$ and $\beta_{j}$ as

$$
\begin{aligned}
\alpha_{1} & =0.5 k_{1} \sqrt{1+\zeta_{1}}, \\
\beta_{1} & =\tau_{1}\left(1+\xi_{1}\right), \\
\alpha_{2} & =0.5 k_{2} \sqrt{1+\zeta_{2}}, \\
\beta_{2} & =\tau_{2}\left(1+\xi_{2}\right),
\end{aligned}
$$

where

$$
\begin{aligned}
\zeta_{1} & =\frac{2\left|W A_{x}^{-}-M A^{-}\right|^{2}}{W^{2}\left(1+A_{3}\right)^{2} \mathbf{A}_{x}^{2}}-1 \\
\zeta_{2} & =\frac{2\left|W\left[\left(1+A_{3}\right)\left(1+B_{3}\right)^{-1} B^{-}\right]_{x}-M\left[\left(1+A_{3}\right)\left(1+B_{3}\right)^{-1} B^{-}\right]\right|^{2}}{W^{2}\left(1+A_{3}\right)^{2} \mathbf{B}_{x}^{2}}-1 \\
\xi_{1} & =\frac{\bar{R}_{x} R-\bar{R} R_{x}-4 i|R|^{2} \nu_{x}}{2 i \alpha_{1}^{2} W^{2}\left(1+A_{3}\right)^{2} \tau_{1}}-1 \\
\xi_{2} & =\frac{\bar{Z}_{x} Z-\bar{Z} Z_{x}-4 i|Z|^{2} \nu_{x}}{2 i \alpha_{2}^{2} W^{2}\left(1+A_{3}\right)^{2} \tau_{2}}-1 .
\end{aligned}
$$

Here

$$
\nu=\partial_{x}^{-1}\left[\frac{A_{1} A_{2 x}-A_{1 x} A_{2}}{\left(1+A_{3}\right) W}-\frac{\left(1+A_{3}\right)\left(B_{1 x} B_{2}-B_{1} B_{2 x}\right)}{\left(1+B_{3}\right)^{2} W}\right] .
$$

We now ready to write the equations for the functions $\alpha_{i}$ and $\beta_{j}$. They satisfy the following four equations

$$
\begin{aligned}
\alpha_{1 t}-2 \alpha_{1 x} \beta_{1}-\alpha_{1} \beta_{1 x} & =0 \\
\beta_{1 t}+\left[\frac{\alpha_{1 x x}}{\alpha_{1}}-\beta_{1}^{2}+2\left(\alpha_{1}^{2}+\alpha_{2}^{2}\right)\right]_{x} & =0 \\
\alpha_{2 t}-2 \alpha_{2 x} \beta_{2}-\alpha_{2} \beta_{2 x} & =0 \\
\beta_{2 t}+\left[\frac{\alpha_{2 x x}}{\alpha_{2}}-\beta_{2}^{2}+2\left(\alpha_{1}^{2}+\alpha_{2}^{2}\right)\right]_{x} & =0 .
\end{aligned}
$$


Let us now we introduce new two complex functions using by the A-transformation. The Atransformation reads as (see e.g. [39- 46])

$$
\begin{aligned}
& q_{1}=\alpha_{1} e^{-i \partial_{x}^{-1} \beta_{1}}, \\
& q_{2}=\alpha_{2} e^{-i \partial_{x}^{-1} \beta_{2}} .
\end{aligned}
$$

Sometime we use the following explicit form of the A-transformation

$$
\begin{aligned}
& q_{1}=0.5 k_{1} \sqrt{1+\zeta_{1}} e^{-i \partial_{x}^{-1}\left[\tau_{1}\left(1+\xi_{1}\right)\right]}, \\
& q_{2}=0.5 k_{2} \sqrt{1+\zeta_{2}} e^{-i \partial_{x}^{-1}\left[\tau_{2}\left(1+\xi_{2}\right)\right]} .
\end{aligned}
$$

It is not difficult to verify that these new complex functions $q_{j}$ satisfy the following system of equations (see e.g. 38])

$$
\begin{aligned}
& i q_{1 t}+q_{1 x x}+2\left(\left|q_{1}\right|^{2}+\left|q_{2}\right|^{2}\right) q_{1}=0 \\
& i q_{2 t}+q_{2 x x}+2\left(\left|q_{1}\right|^{2}+\left|q_{2}\right|^{2}\right) q_{2}=0
\end{aligned}
$$

It is nothing but the Manakov system. So we proved that the Manakov system (3.52)-(3.53) is the Lakshmanan equivalent counterpart of the 2-layer M-LIII equation (2.10)-(2.11) or in the vector form (3.1)-(3.2). Finally we note that if $\zeta_{j}=\xi_{j}=0$ then the A-transformation (3.48)-(3.49) or (3.50)-(3.51) reduces to the Hasimoto transformation

$$
\begin{aligned}
& q_{1}=0.5 \kappa_{1} e^{-i \partial_{x}^{-1} \tau_{1}}, \\
& q_{2}=0.5 \kappa_{2} e^{-i \partial_{x}^{-1} \tau_{2}} .
\end{aligned}
$$

\section{Lax representation of the coupled M-LIII equation hier- archy}

In the previous section we have shown that the coupled M-LIII equation is the Lakshmanan equivalent to the Manakov system. This means that the coupled M-LIII equation is integrable by the IST method since its equivalent counterpart - the Manakov system is integrable. In turn, it means that the M-LIII equation admits all ingredients of integrable systems like Lax representation (LR), infinite number of commuting integrals of motion, n-soliton solutions etc. Below we present two possible versions of the LR for the M-LIII equation hierarchy.

\subsection{LR type - I}

The first type of LR for the coupled M-LIII equation hierarchy reads as

$$
\begin{aligned}
Y_{x} & =-i \lambda P Y, \\
Y_{t} & =\sum_{j=1}^{N} \lambda^{j} V_{j} Y,
\end{aligned}
$$

where $\lambda$ is a spectral parameter and

$$
P=\frac{1}{2+K}\left(\begin{array}{ccc}
2 A_{3}-K & 2 A^{-} & \frac{2\left(1+A_{3}\right) B^{-}}{1+B_{3}} \\
2 A^{+} & -\left(2 A_{3}+K\right) & \frac{2 A^{+} B^{-}}{1+B_{3}} \\
\frac{2\left(1+A_{3}\right) B^{+}}{1+B_{3}} & \frac{2 A^{-} B^{+}}{1+B_{3}} & K-2
\end{array}\right),
$$

with $K=\left(1+A_{3}\right)\left(1-B_{3}\right)\left(1+B_{3}\right)^{-1}$. The compatibility condition of this system gives the coupled M-LIII equation hierarchy. As the particular example, let us consider the case when $N=2$. Then the set of equations (4.1)-(4.2) takes the form

$$
\begin{aligned}
Y_{x} & =-i \lambda P Y, \\
Y_{t} & =\left(\lambda^{2} V_{2}+\lambda V_{1}\right) Y,
\end{aligned}
$$


where

$$
V_{2}=-2 i P, \quad V_{1}=P P_{x}
$$

The compatibility condition of the equations (4.4)-(4.5) gives the 2-layer M-LIII equation (2.10)(2.11) or (3.1)-(3.2) that is same.

\subsection{LR type - II}

The second type of LR for the coupled M-LIII equation hierarchy can be written in the following form

$$
\begin{aligned}
Y_{x} & =-i \lambda Q Y, \\
Y_{t} & =\sum_{j=1}^{N} \lambda^{j} W_{j} Y .
\end{aligned}
$$

Here

$$
Q=Q_{1}+Q_{2}
$$

where

$$
Q_{1}=\left(\begin{array}{cccc}
0 & A_{1} & A_{2} & A_{3} \\
-A_{1} & 0 & A_{3} & -A_{2} \\
-A_{2} & -A_{3} & 0 & A_{1} \\
-A_{3} & A_{2} & -A_{1} & 0
\end{array}\right), \quad Q_{2}=\left(\begin{array}{cccc}
0 & B_{1} & B_{2} & -B_{3} \\
-B_{1} & 0 & B_{3} & B_{2} \\
-B_{2} & -B_{3} & 0 & -B_{1} \\
B_{3} & -B_{2} & B_{1} & 0
\end{array}\right)
$$

From the compatibility condition of the set of equations (4.7)-(4.8) $Y_{x t}=Y_{t x}$ we obtain the coupled M-LIII equation hierarchy.

\section{Relation between solutions of the coupled M-LIII equa- tion and the Manakov system}

Let $A_{j}$ and $B_{j}$ be the solution of the coupled M-LIII equation (2.10)-(2.11). Then the solution of the Manakov system (3.52)-(3.53) is given by

$$
\begin{aligned}
q_{1} & =\frac{R e^{2 i \nu}}{W\left(1+A_{3}\right)}, \\
q_{2} & =\frac{Z e^{2 i \nu}}{W\left(1+A_{3}\right)} .
\end{aligned}
$$

\section{Gauge equivalence between the $\Gamma$-spin system and the Manakov system}

Above, we have proved that the coupled M-LIII equation (2.10)-(2.11) and the Manakov system (3.52)-(3.53) is the Lakshmanan equivalent to each other. In this section, we want to present the another (gauge) equivalent counterpart of the Manakov system. It is well-known that the Lax representation of the Manakov equation (3.52)-(3.53) has the form (see e.g. 38])

$$
\begin{aligned}
& \Phi_{x}=U \Phi, \\
& \Phi_{t}=V \Phi .
\end{aligned}
$$

Here

$$
U=-i \lambda \Sigma+U_{0}, \quad V=-2 i \lambda^{2} \Sigma+2 \lambda U_{0}+V_{0}
$$


with

$$
\Sigma=\left(\begin{array}{ccc}
1 & 0 & 0 \\
0 & -1 & 0 \\
0 & 0 & -1
\end{array}\right), U_{0}=\left(\begin{array}{ccc}
0 & q_{1} & q_{2} \\
-\bar{q}_{1} & 0 & 0 \\
-\bar{q}_{2} & 0 & 0
\end{array}\right), V_{0}=i\left(\begin{array}{ccc}
\left|q_{1}\right|^{2}+\left|q_{2}\right|^{2} & q_{1 x} & q_{2 x} \\
\bar{q}_{1 x} & -\left|q_{1}\right|^{2} & -\bar{q}_{1} q_{2} \\
\bar{q}_{2 x} & -\bar{q}_{2} q_{1} & -\left|q_{2}\right|^{2}
\end{array}\right) .
$$

Let us now consider the gauge transformation

$$
\Psi=g^{-1} \Phi, \quad g=\Phi_{\lambda=0} .
$$

Then $\Psi$ obeys the equations

$$
\begin{aligned}
& \Psi_{x}=U^{\prime} \Psi \\
& \Psi_{t}=V^{\prime} \Psi
\end{aligned}
$$

where

$$
U^{\prime}=-i \lambda \Gamma, \quad V^{\prime}=-2 i \lambda^{2} \Gamma+\frac{1}{2} \lambda\left[\Gamma, \Gamma_{x}\right] .
$$

Here

$$
\Gamma=g^{-1} \Sigma g, \quad \Gamma^{2}=I
$$

and

$$
\Gamma=\left(\begin{array}{lll}
\Gamma_{11} & \Gamma_{12} & \Gamma_{13} \\
\Gamma_{21} & \Gamma_{22} & \Gamma_{23} \\
\Gamma_{31} & \Gamma_{32} & \Gamma_{33}
\end{array}\right)
$$

Elements of the $\Gamma$ matrix satisfy some restrictions

$$
\Gamma_{33}=-\left(1+\Gamma_{11}+\Gamma_{22}\right), \quad \Gamma_{i j}=\bar{\Gamma}_{j i},
$$

and

$$
\begin{array}{ll}
\Gamma_{i k} \Gamma_{k j}+\Gamma_{i(k+1)} \Gamma_{(k+1) i}+\Gamma_{i(k+2)} \Gamma_{(k+2) i} & =0,(i \neq k \neq j), \\
\Gamma_{i k} \Gamma_{k i}+\Gamma_{i(k+1)} \Gamma_{(k+1) i}+\Gamma_{i(k+2)} \Gamma_{(k+2) i} & =1 .
\end{array}
$$

The compatibility condition of the equations (5.6)-(5.7) gives

$$
i \Gamma_{t}+\frac{1}{2}\left[\Gamma, \Gamma_{x x}\right]=0 .
$$

We call this equation - the $\Gamma$-spin system. Thus the $\Gamma$-spin system (6.14) is the gauge equivalent counterpart of the Manakov system (3.52)-(3.53). It is the well-known result (see e.g. [38). In terms of elements, the $\Gamma$-spin system (6.14) reads as

$$
\begin{aligned}
i \Gamma_{11 t}+\frac{1}{2}\left(\Gamma_{12} \Gamma_{21 x x}+\Gamma_{13} \Gamma_{31 x x}-\Gamma_{12 x x} \Gamma_{21}-\Gamma_{13 x x} \Gamma_{31}\right) & =0,(6.15) \\
i \Gamma_{12 t}+\frac{1}{2}\left(\Gamma_{11} \Gamma_{12 x x}+\Gamma_{12} \Gamma_{22 x x}+\Gamma_{13} \Gamma_{32 x x}-\Gamma_{11 x x} \Gamma_{12}-\Gamma_{12 x x} \Gamma_{22}-\Gamma_{13 x x} \Gamma_{32}\right) & =0,(6.16) \\
i \Gamma_{13 t}+\frac{1}{2}\left(\Gamma_{11} \Gamma_{13 x x}+\Gamma_{12} \Gamma_{23 x x}+\Gamma_{13} \Gamma_{33 x x}-\Gamma_{11 x x} \Gamma_{13}-\Gamma_{12 x x} \Gamma_{23}-\Gamma_{11 x x} \Gamma_{13}\right) & =0,(6.17) \\
i \Gamma_{21 t}+\frac{1}{2}\left(\Gamma_{21} \Gamma_{11 x x}+\Gamma_{22} \Gamma_{21 x x}+\Gamma_{23} \Gamma_{31 x x}-\Gamma_{21 x x} \Gamma_{11}-\Gamma_{22 x x} \Gamma_{21}-\Gamma_{23 x x} \Gamma_{31}\right) & =0,(6.18) \\
i \Gamma_{22 t}+\frac{1}{2}\left(\Gamma_{21} \Gamma_{12 x x}+\Gamma_{23} \Gamma_{32 x x}-\Gamma_{21 x x} \Gamma_{12}-\Gamma_{23 x x} \Gamma_{32}\right) & =0,(6.19) \\
i \Gamma_{23 t}+\frac{1}{2}\left(\Gamma_{21} \Gamma_{13 x x}+\Gamma_{22} \Gamma_{23 x x}+\Gamma_{23} \Gamma_{33 x x}-\Gamma_{21 x x} \Gamma_{13}-\Gamma_{22 x x} \Gamma_{23}-\Gamma_{23 x x} \Gamma_{33}\right) & =0,(6.20) \\
i \Gamma_{31 t}+\frac{1}{2}\left(\Gamma_{31} \Gamma_{11 x x}+\Gamma_{32} \Gamma_{21 x x}+\Gamma_{33} \Gamma_{31 x x}-\Gamma_{31 x x} \Gamma_{11}-\Gamma_{32 x x} \Gamma_{21}-\Gamma_{33 x x} \Gamma_{31}\right) & =0,(6.21) \\
i \Gamma_{32 t}+\frac{1}{2}\left(\Gamma_{31} \Gamma_{12 x x}+\Gamma_{32} \Gamma_{22 x x}+\Gamma_{33} \Gamma_{32 x x}-\Gamma_{31 x x} \Gamma_{12}-\Gamma_{32 x x} \Gamma_{22}-\Gamma_{33 x x} \Gamma_{32}\right) & =0,(6.22) \\
i \Gamma_{33 t}+\frac{1}{2}\left(\Gamma_{31} \Gamma_{13 x x}+\Gamma_{32} \Gamma_{23 x x}-\Gamma_{31 x x} \Gamma_{13}-\Gamma_{32 x x} \Gamma_{23}\right) & =0 .(6.23)
\end{aligned}
$$




\section{Relation between solutions of the coupled M-LIII equa- tion and the $\Gamma$-spin system}

In the previous sections we have shown that to the one and same set of equations - the Manakov system (3.52)-(3.53), correspond two spin systems: the coupled M-LIII equation (3.1)-(3.2) and the $\Gamma$-spin system (6.14). It tells us that between these two spin systems there must be some exact relation/correspondence. In other words, the 2-layer M-LIII equation (2.10)-(2.11) and the $\Gamma$-spin system (6.14) are equivalent to each other by some exact transformations. Below we will present these transformations.

\subsection{Direct M-transformation}

According to the M-transformation, in terms of the spin vectors $\mathbf{A}$ and $\mathbf{B}$, the elements of the $\Gamma$-spin system are expressed as

$$
\Gamma=\frac{1}{2+K}\left(\begin{array}{ccc}
2 A_{3}-K & 2 A^{-} & \frac{2\left(1+A_{3}\right) B^{-}}{1+B_{3}} \\
2 A^{+} & -\left(2 A_{3}+K\right) & \frac{2 A^{+} B^{-}}{1+B_{3}} \\
\frac{2\left(1+A_{3}\right) B^{+}}{1+B_{3}} & \frac{2 A^{-} B^{+}}{1+B_{3}} & K-2
\end{array}\right)
$$

where

$$
K=\frac{\left(1+A_{3}\right)\left(1-B_{3}\right)}{1+B_{3}}
$$

This is the direct M-transformation. This M-transformation allows us to find solutions of the $\Gamma$-spin system (6.14) if we know the solutions of the coupled M-LIII equation (2.10)-(2.11).

\subsection{Inverse M-transformation}

According to the inverse M-transformation, solutions of the coupled M-LIII equation can be expressed by the components of the $\Gamma$-spin system as

$$
\begin{aligned}
A & =\frac{1}{1-\Gamma_{33}}\left(\begin{array}{cc}
\Gamma_{11}-\Gamma_{22} & 2 \Gamma_{12} \\
2 \Gamma_{21} & \Gamma_{22}-\Gamma_{11}
\end{array}\right), \\
B & =\frac{1}{1-\Gamma_{22}}\left(\begin{array}{cc}
\Gamma_{11}-\Gamma_{33} & 2 \Gamma_{13} \\
2 \Gamma_{31} & \Gamma_{33}-\Gamma_{11}
\end{array}\right) .
\end{aligned}
$$

The transformations (7.3)-(7.4) is called the inverse M-transformation. Using the inverse Mtransformation, we can find solutions of the coupled M-LIII equation (2.10)-(2.11), if we know the solutions of the $\Gamma$-spin system (6.14).

\section{Conclusions}

In this paper, we have shown how the dynamics of two interacting and moving curves in some space $R^{n}$ can be related to the dynamics of the coupled spin systems, namely, the coupled M-LIII equation. Next, after some algebra we have proved that these two interacting and moving curves are related with the Manakov system. On the other hand, it is well-known that the Manakov system is equivalent to the $\Gamma$-spin system. Also, we have presented the transformations which established the relation between solutions of the $\Gamma$-spin system and the coupled M-LIII equation. Our results can also be generalized to higher dimensional spaces (see e.g. refs. [39]-[46]). Work along these lines is in progress. 


\section{References}

[1] Gutshabash E. Sh. Zapiski nauchnyh seminarov POMI, 269, 164-179 (2000)

[2] Nian-Ning Huang, Bing Xu. Commun. Theor. Phys., 12, 121-126 1989).

[3] G. Nugmanova, Z. Zhunussova, K. Yesmakhanova, G. Mamyrbekova, R. Myrzakulov. International Journal of Mathematical, Computational, Statistical, Natural and Physical Engineering, 9, N8, 328-331 (2015).

[4] J.-S. He, Y. Cheng, Y.-S. Li. Commun. Theor. Phys., 38, 493-496 (2002).

[5] U. Saleem, M. Hasan. J. Phys. A: Math. Theor., 43, 045204 (2010).

[6] M. Lakshmanan, Phil. Trans. R. Soc. A, 369 1280-1300 (2011).

[7] M. Lakshmanan, Phys. Lett. A, 64, 53-54 (1977).

[8] L.A. Takhtajan, Phys. Lett. A, 64, 235-238 (1977).

[9] C. Senthilkumar, M. Lakshmanan, B. Grammaticos, A. Ramani, Phys. Lett. A 356 339-345 (2006).

[10] Y. Ishimori, Prog. Theor. Phys. 7233 (1984).

[11] R. Myrzakulov, S. Vijayalakshmi, G. Nugmanova, M. Lakshmanan Physics Letters A, 233 , 14-6, 391-396 (1997).

[12] R. Myrzakulov, S. Vijayalakshmi, R. Syzdykova, M. Lakshmanan, J. Math. Phys., 39, 21222139 (1998).

[13] R. Myrzakulov, M. Lakshmanan, S. Vijayalakshmi, A. Danlybaeva , J. Math. Phys., 39, 3765-3771 (1998).

[14] Myrzakulov R, Danlybaeva A.K, Nugmanova G.N. Theoretical and Mathematical Physics, V.118, 13, P. 441-451 (1999).

[15] Myrzakulov R., Nugmanova G., Syzdykova R. Journal of Physics A: Mathematical \& Theoretical, V.31, 147, P.9535-9545 (1998).

[16] Myrzakulov R., Daniel M., Amuda R. Physica A., V.234, 13-4, P.715-724 (1997).

[17] Myrzakulov R., Makhankov V.G., Pashaev O.. Letters in Mathematical Physics, V.16, N1, P.83-92 (1989)

[18] Myrzakulov R., Makhankov V.G., Makhankov A. Physica Scripta, V.35, N3, P. 233-237 (1987)

[19] Myrzakulov R., Pashaev O.., Kholmurodov Kh. Physica Scripta, V.33, N4, P. 378-384 (1986)

[20] Anco S.C., Myrzakulov R. Journal of Geometry and Physics, v.60, 1576-1603 (2010)

[21] Myrzakulov R., Rahimov F.K., Myrzakul K., Serikbaev N.S. On the geometry of stationary Heisenberg ferromagnets. In: "Non-linear waves: Classical and Quantum Aspects", Kluwer Academic Publishers, Dordrecht, Netherlands, P. 543-549 (2004)

[22] Myrzakulov R., Serikbaev N.S., Myrzakul Kur., Rahimov F.K. On continuous limits of some generalized compressible Heisenberg spin chains. Journal of NATO Science Series II. Mathematics, Physics and Chemistry, V 153, P. 535-542 (2004)

[23] R.Myrzakulov, G. K. Mamyrbekova, G. N. Nugmanova, M. Lakshmanan. Symmetry, 7(3), 1352-1375 (2015). arXiv:1305.0098

[24] R.Myrzakulov, G. K. Mamyrbekova, G. N. Nugmanova, K. Yesmakhanova, M. Lakshmanan. Physics Letters A, 378, N30-31, 2118-2123 (2014). arXiv:1404.2088. 
[25] Myrzakulov R., Martina L., Kozhamkulov T.A., Myrzakul Kur. Integrable Heisenberg ferromagnets and soliton geometry of curves and surfaces. In book: "Nonlinear Physics: Theory and Experiment. II". World Scientific, London, P. 248-253 (2003)

[26] Myrzakulov R. Integrability of the Gauss-Codazzi-Mainardi equation in 2+1 dimensions. In "Mathematical Problems of Nonlinear Dynamics", Proc. of the Int. Conf. "Progress in Nonlinear sciences", Nizhny Novgorod, Russia, July 2-6, 2001, V.1, P.314-319 (2001)

[27] Chen Chi, Zhou Zi-Xiang. Darboux Tranformation and Exact Solutions of the Myrzakulov-I Equations. Chin. Phys. Lett., 26, N8, 080504 (2009)

[28] Chen Hai, Zhou Zi-Xiang. Darboux Transformation with a Double Spectral Parameter for the Myrzakulov-I Equation. Chin. Phys. Lett., 31, N12, 120504 (2014)

[29] Zhao-Wen Yan, Min-Ru Chen, Ke Wu, Wei-Zhong Zhao. J. Phys. Soc. Jpn., 81, 094006 (2012)

[30] Yan Zhao-Wen, Chen Min-Ru, Wu Ke, Zhao Wei-Zhong. Commun. Theor. Phys., 58, 463-468 (2012)

[31] K.R. Ysmakhanova, G.N. Nugmanova, Wei-Zhong Zhao, Ke Wu. Integrable inhomogeneous Lakshmanan-Myrzakulov equation, nlin/0604034

[32] Zhen-Huan Zhang, Ming Deng, Wei-Zhong Zhao, Ke Wu. On the integrable inhomogeneous Myrzakulov-I equation, [arXiv: nlin/0603069

[33] Martina L, Myrzakul Kur., Myrzakulov R, Soliani G. Journal of Mathematical Physics, V.42, 13, P.1397-1417 (2001).

[34] Xiao-Yu Wu, Bo Tian, Hui-Ling Zhen, Wen-Rong Sun and Ya Sun. Journal of Modern Optics, 2015.

[35] Z.S. Yersultanova, M. Zhassybayeva, K. Yesmakhanova, G. Nugmanova, R. Myrzakulov. International Journal of Geometric Methods in Modern Physics, 13, N1, 1550134 (2016).. arXiv:1404.2270,

[36] Bordag L.A., Yanovski A.B. J. Phys. A: Math. Gen., 28, 4007-4013 (1995)

[37] Bordag L.A., Yanovski A.B. J. Phys. A: Math. Gen., 29, 5575-5590 (1996)

[38] N. A. Kostov, R. Dandoloff, V. S. Gerdjikov, G. G. Grahovski. The Manakov system as two moving interacting curves, In the Proceedings of the International Workshop "Complex structures and vector fields", August 21-26, 2006, Sofia, Bulgaria. Eds.: K. Sekigawa, S. Dimiev. World Scientific (2007)

[39] Myrzakul Akbota. Integrability of two coupled curves and geometrically equivalent spin analogue of the Manakov equation. Vestnik ENU, N2, 95-99 (2016)

[40] Myrzakul Akbota. Equivalence between the coupled M-LIII equation and the $\Gamma$-spin system. Vestnik ENU, N3, 89-94 (2016)

[41] Myrzakul Akbota. Gauge equivalence between the coupled M-LIII equation and the Manakov system. Vestnik ENU, N1, 54-60 (2016)

[42] Myrzakul Akbota and Myrzakulov Ratbay. Motion of two interacting curves and surfaces: integrable reductions and soliton equations. Vestnik ENU, N4, 45-57 (2014)

[43] Myrzakul Akbota and Myrzakulov Ratbay. Integrable Motion of Two Interacting Curves and Heisenberg Ferromagnetic Equations, Abstracts of XVIII-th Intern. Conference "Geometry, Integrability and Quantization", June 3-8, 2016, Bulgaria.

[44] Myrzakul Akbota and Myrzakulov Ratbay. Integrable motion of two interacting curves, spin systems and the Manakov system, arXiv:1606.06598 
[45] Myrzakul Akbota and Myrzakulov Ratbay. Darboux transformations and exact soliton solutions of integrable coupled spin systems related with the Manakov system, arXiv:1607.08151]

[46] Myrzakul Akbota and Myrzakulov Ratbay. Integrable geometric flows of interacting curves/surfaces, multilayer spin systems and the vector nonlinear Schrodinger equation, arXiv:1608.08553, 